\title{
Genomes From Verteba Cave Suggest Diversity Within The Trypillians In Ukraine
}

Pere Gelabert ( $\sim$ pere.gelabert@univie.ac.act )

University of Vienna

Ryan W. Schmidt

University of Porto

Daniel M. Fernandes

University of Vienna

Jordan K. Karsten

University of Wisconsin-Oshkosh

\section{Thomas K. Harper}

The Pennsylvania State University

\section{Gwyn D. Madden}

Grand Valley State University

\section{Sarah H. Ledogar}

University of New England

Mykhailo Sokhatsky

Borschiv Regional Museum of Local Lore

Hiroki Oota

University of Tokyo

\section{Douglas j. Kennett}

University of California, Santa Barbara

\section{Ron Pinhasi}

University of Vienna

\section{Research Article}

Keywords: transition, agriculture, process, hunter

Posted Date: November 12th, 2021

DOI: https://doi.org/10.21203/rs.3.rs-1044480/v1

License: (c) (i) This work is licensed under a Creative Commons Attribution 4.0 International License. Read Full License 


\section{Abstract}

The transition to agriculture occurred relatively late in Eastern Europe, leading researchers to debate whether it was a gradual, interactive process or a colonization event. In the forest and forest-steppe regions of Ukraine, farming appeared during the fifth millennium BCE, associated with the Cucuteni-Trypillian Archaeological Complex (CTCC, 4800-3000 BCE). Across Europe, the Neolithization process was highly variable across space and over time. Here, we investigate the population dynamics of early agriculturalists from the eastern forest-steppe region based on analyses of 20 ancient genomes from the Verteba Cave site (3789-980 BCE). The results reveal that the CTCC individuals' ancestry is related to both western hunter gatherers and Near Eastern farmers, lacks local ancestry associated with Ukrainian Neolithic hunter gatherers and has steppe ancestry. An Early Bronze Age individual has an ancestry profile related to the Yamnaya expansions but with $20 \%$ ancestry related to the other Trypillian individuals, which suggests admixture between the Trypillians and the incoming populations carrying steppe-related ancestry. A Late Bronze Age individual dated to 980-948 BCE has a genetic profile indicating affinity to Beaker-related populations, detected close to 1,000 years after the end of the Bell Beaker phenomenon during the Third millennium BCE.

\section{Introduction}

The Neolithization process in Europe resulted in dramatic technological and cultural shifts, which included novel subsistence practices ${ }^{1}$. There are two major groups of models that explain the Neolithization process: demic diffusion models describe Neolithization as a colonization process by farmers that is propelled by exponential population growth characteristic of the Neolithic, whereas acculturation models outline the process as one in which at least some of the transition entails indigenous hunting-foraging groups that adopt farming following periods of variable length during which they interact with neighbouring exogenous farmers ${ }^{2}$. Across most of Europe, the Neolithic transition was genetically defined by profound population replacement, consistent with the demic diffusion of people from Anatolia ${ }^{3-5}$. The Anatolian farmers reached the Balkans and other regions of Southeast Europe in the 7th millennium BCE ${ }^{6,7}$ and subsequently spread further via the Mediterranean and later through the Danube, substantially replacing indigenous Mesolithic European populations ${ }^{8,9}$

In contrast to central Europe ${ }^{10,11}$, areas of Eastern Europe, including Ukraine, Moldova, Western Russia, and Romania, did not adopt agriculture until the late Neolithic ( 4,500 BCE), although various sedentary and semisedentary huntergatherer Mesolithic groups in these regions began using pottery as early as $8,500 \mathrm{BCE}^{12,13}$.

The Cucuteni-Trypillia cultural complex (CTCC) is a grouping of several interrelated Middle Neolithic/Eneolithic cultures in parts of Ukraine, Moldova, and Romania ${ }^{14,15}$. This complex stretches from the Transylvanian Alps to the Dnieper River and is named for the type sites of Cucuteni in lași County, Romania and Trypillia (also known as Tripolye, in Russian) in the Kyiv oblast, Ukraine. The Cucuteni and Trypillia cultures have common roots in the Precucuteni culture; the earliest CTCC sites are found in the piedmont of the Carpathian Mountains, and the earliest radiocarbon dates (from the Precucuteni 2 period) date to approximately 4800 BCE ${ }^{16,17}$. The CTCC originated from the interaction of several Danubian Neolithic groups, with evidence for similarities in house construction, ceramic styles, and lithic artifact production $^{10,16,18,19}$.

Following the origin of this cultural complex in the Carpathian piedmont, the CTCC eventually occupied a territory covering much of the modern territories of Ukraine, Moldova, and Romania. The first diagnostically early Trypillia (Trypillia A) sites diverged from the Precucuteni culture 4500 BCE in the Dniester River valley. Later, population movements, occurring from the middle period (Trypillia B1) onward, saw that the Trypillia culture expanded to Volhynia in the west and the Dniepr River in the east. This territorial expansion is believed to have resulted primarily from demographic increases associated with a successful agropastoral subsistence strategy and the search for new arable 
land for cultivation ${ }^{18}$. However, some population growth may have been the product of Trypillian populations incorporating indigenous hunting and gathering $(\mathrm{HG})$ groups, such as members of the Bug-Dneister culture. Another mode of population increase could have been the acculturation of refugees following the collapse of the Neolithic in Romania, Hungary, and Bulgaria. During the middle-to-late periods of the Trypillia culture (Trypillia B2 to C1-2; 41003400 BCE), some CTCC groups established extremely large settlements in Central Ukraine, often referred to as "giant settlements" or "megasites", which attained sizes of 100-320 ha ${ }^{20}$. Rapid demographic growth within the CTCC around the turn of the fourth millennium BCE necessitated the exploitation of new territories, precipitating migrations to previously peripheral areas $^{21}$.

Hypotheses for the rise of megasites in particular are varied; it has been suggested that they may have been a defensive response to threats posed by steppe pastoralists or competing subgroups within the CTCC 22,23 or that they simply represent ephemeral episodes of population agglomeration due to large-scale migration from the Dniester region ${ }^{24}$.

Even though Trypillian populations established a high density of settlements in western and central Ukraine ${ }^{25}$, very few burials have been located. Only a handful of cemeteries dating to the Late Trypillia period were excavated during the 1960s and 1970s, such as Chapaievka in Ukraine ${ }^{26}$ and Vykhvatyntsi in Moldova ${ }^{27}$. While these sites give some glimpse into Trypillian mortuary behavior, they are limited in their temporal scope and have not been subjected to modern laboratory analyses. To better understand the origins, connections, and diversity of the CTCC, we collected human remains from three chambers at the Verteba Cave (VC) site in the Ternopil Oblast, Ukraine, one of the few sites that contain human remains associated with the CTCC (Fig. 1A). Accelerator mass spectrometry (AMS) ${ }^{14} \mathrm{C}$ dates of humans

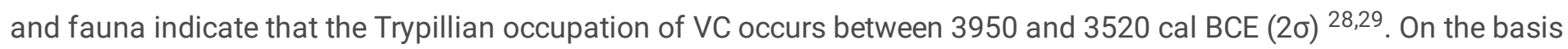
of the ceramic assemblages present in the cave and a sample of lower-resolution liquid scintillation ${ }^{14} \mathrm{C}$ dates, it is probable that occupation continued for some time into the Late Trypillia (C2) period and Early Bronze Age transition ${ }^{30}$. More recently, AMS radiocarbon dating has also identified deposits at different locations throughout the cave dating to the Mesolithic (7950-7490 cal BCE [2б]), Bronze Age, Iron Age and Medieval period ${ }^{31}$. Skeletal assemblages were taken from three separate chambers (Site 7, Site 17, and Site 20) of the cave (Table S1, Fig. 1B). Each of these chambers contains CTCC material culture; however, burials in the cave are secondary in nature, and disturbance through human activity during antiquity and bioturbation complicate reconstruction of the cave's use and chronology (Table S9). Most individuals in this study came from Site 7, which has been extensively documented through ceramic analysis and radiocarbon dating ${ }^{31,32}$, with peak occupation dated to periods $\mathrm{C} 1$ and C1-2 of Trypillia periodization ( 3,900-3,350 $\mathrm{BCE})$. Interpretations regarding the use of the cave are varied, including its use as a temporary shelter, ritual site or mortuary location $29,33,34$. There is additional evidence to support the idea that the burials in the cave, which are largely commingled and secondary in nature, are representative of victims of warfare or sacrifice ${ }^{35}$.

The paleogenetics of the Trypillian population is limited to the analysis of uniparental markers (mtDNA) and genomewide analysis of 8 individuals. Mitochondrial haplogroups typical of ancient Eurasian farming groups $(H, H V, T, K, J)$ have been observed for these individuals scattered throughout the cave $\mathrm{e}^{30,32,36}$. Schmidt et al. (2020) found withinchamber evidence for haplogroup W, which has been observed for steppe populations associated with the Corded Ware and Unetice cultures of the middle Volga region ${ }^{8}$. Genome-wide analyses of CTCC individuals have shown ancestral components predominantly of Early Neolithic farming groups (estimated to be 60-80\%), confirming that the early farmers who settled Western and Central Ukraine were largely derived from the same source population as the farmers of Anatolia and Western Europe ${ }^{13,37}$. The remaining 20 percent of their ancestry is less certain. Mathieson et al. (2018) found that this ancestral component was a mix of western (WHG) and eastern (EHG) hunter-gatherers (HG) found in HG groups inhabiting the region during the Neolithic. Immel et al. (2020) recovered genome-wide data from four individuals buried at two separate sites in northern Moldova that date to 3,500 - 3,100 cal BCE (during the Late Trypillia period), five 
centuries younger than the datations in Verteba cave, and found a larger degree of steppe-related ancestry (albeit in varying proportions among the sampled individuals). This observation may be explained by the gradual assimilation of local Mesolithic and Neolithic HG groups into the Trypillian population, at least for groups who settled in Moldova.

The settlement systems of the Trypillia culture interacted with both Central European and steppe populations. Archaeological evidence for steppe interactions is found in shell-tempered pottery, which is similar to steppe-style wares 35,38. Some of these look nearly identical to pottery found at steppe sites, while others combine shell tempering with CTCC decorative motifs ${ }^{35}$. Symbolic objects influenced by-or directly imported from-steppe communities, such as stone mace heads, are found at some Middle-to-Late Trypillia sites ${ }^{35}$, and exchanges of pottery are evident as early as Trypillia B2 ${ }^{39}$. There was undoubtedly some degree of interaction between Trypillian populations and the Dnieper-Donets culture, while any synchronicity between the Trypillia culture and the following Yamnaya horizon was likely very brief. Regardless, however, some Trypillian populations were likely in permanent contact with steppe populations ${ }^{40}$. Interestingly, after approximately 3,400 BCE, the Trypillian mega-sites were largely abandoned. The cause of this abandonment has been widely debated. One hypothesis is an increased conflictivity with the steppe populations once these populations started to expand westwards. Such a hypothesis is based on the frequent warfare discovered in Verteba cave ${ }^{23,35}$.

Here, we recovered genome-wide sequence data from 20 individuals buried in VC, eleven of which were directly dated by AMS ${ }^{14} \mathrm{C}$ to the interval of 3,930 to $980 \mathrm{cal} \mathrm{BCE}$. We use these data to specifically test several questions: (1) Is there evidence for admixture with local HGs, as has been suggested by Rascovan et al. ${ }^{29}$; (2) using our expanded dataset with higher coverage than in Mathieson et al. ${ }^{13}$, can we clarify the Neolithic ancestral component of the Trypillian population, i.e., can we show that they are more similar to early farmers from Anatolia, the LBK (Linearbandkeramik), or elsewhere; (3) since the CTCC individuals lived in close proximity to steppe populations, is there evidence for genetic admixture with the Yamnaya or earlier steppe populations? (4) Do later Bronze Age populations that settled in the region share genetic affinities with the CTCC group from verteba cave?

\section{Materials And Methods}

To perform the present study, 23 samples were collected from Verteba cave (Ukraine). Due to low coverage, two samples were not included in the final analyses. A complete description of the methods can be found in the Supplementary Information section.

\section{Ams Radiocarbon Analysis}

The ages of individuals from this study were determined using AMS ${ }^{14} \mathrm{C}$ dating; here, we report five new dates run at the Pennsylvania State University Accelerator Mass Spectrometry Laboratory (PSUAMS) along with three existing dates from the Oxford Accelerator Unit (OxA) and Beta Analytic (Beta) (Lillie et al. 2017; Mathieson et al. 2018; see Supplementary Table 1).

Bone collagen extraction for ${ }^{14} \mathrm{C}$ and stable isotope analysis was extracted and purified at Pennsylvania State University using a modified Longin method with ultrafiltration ${ }^{60}$. In cases where this method returned an unacceptably low gelatin yield, samples were processed according to the XAD amino acid purification method ${ }^{61}$. Samples were prepared first by manual cleaning adhering sediment and removing exposed surfaces with an X-acto blade. This was followed by demineralization for $24-36$ hours in $0.5 \mathrm{~N} \mathrm{HCl}$ at $5^{\circ} \mathrm{C}$. The pseudomorph was then rinsed to neutrality in multiple changes of Nanopure $\mathrm{H}_{2} \mathrm{O}$ before being gelatinized for 10 hours at $60^{\circ} \mathrm{C}$ in 0.01 The resulting gelatin was lyophilized, visually inspected and then weighed to assess bone collagen preservation. Rehydrated gelatin solution was pipetted into 
precleaned Centriprep ${ }^{62}$ ultrafilters (retaining $30 \mathrm{kDa}$ molecular weight gelatin), centrifuged 3 times for 20 minutes, diluted with Nanopure $\mathrm{H}_{2} \mathrm{O}$, and centrifuged 3 more times for 20 minutes to desalt the solution. Carbon and nitrogen concentrations and stable isotope ratios were measured at the Yale Analytical and Stable Isotope Center with a Costech elemental analyser (ECS 4010) and Thermo DeltaPlus analyser. Sample quality was evaluated by examining the \% crude gelatin yield, \%,$\% \mathrm{~N}$ and $\mathrm{C}: \mathrm{N}$ ratios before $\mathrm{AMS}{ }^{14} \mathrm{C}$ dating. $\mathrm{C}: \mathrm{N}$ ratios for the dated samples fell between 3.15 and 3.42 , indicating acceptable collagen preservation ${ }^{63}$. Collagen samples were combusted for three hours at $900^{\circ} \mathrm{C}$ in vacuumsealed quartz tubes with $\mathrm{CuO}$ and Ag wires. Sample $\mathrm{CO} 2$ was reduced to graphite at $550^{\circ} \mathrm{C}$ using $\mathrm{H} 2$ and an Fe catalyst, with reaction water drawn off with $\mathrm{Mg}\left(\mathrm{ClO}_{4}\right)_{2}{ }^{64}$. Graphite samples were pressed into targets in $\mathrm{Al}$ cathodes and loaded on the target wheel for AMS analysis. The ${ }^{14} \mathrm{C}$ ages were corrected for mass-dependent fractionation with measured $\delta^{13} \mathrm{C}$ values ${ }^{65}$ and compared with samples of Pleistocene whale bone (backgrounds, $48,000{ }^{14} \mathrm{C} \mathrm{BP}$ ), late Holocene bison bone $\left(\sim 1,850{ }^{14} \mathrm{C} B P\right)$, late $A D 1800$ s cow bone and OX-2 oxalic acid standards for calibration.

Dates were calibrated according to the IntCal13 Northern Hemisphere calibration curve ${ }^{66}$ using OxCal $4.3^{67}$. Seven of the

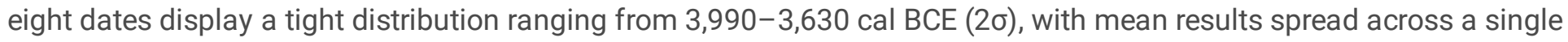
century from 3,770-3,670 cal BCE. These results are comparable with dates from other sites in Romania, Moldova and Ukraine dating to the period Trypillia C1 and are consistent with the Trypillia C1 (Shypenetskaya local group) occupation identified at the site (Nikitin et al. 2010). A single date corresponds with the Middle Bronze Age (PSUAMS-3153; VERT$113)$, dating to the range of $1960-1770 \mathrm{cal}$ BCE $(2 \sigma)$.

\section{Dna Extraction And Library Preparation}

All laboratory work was performed in dedicated ancient DNA laboratories at University College, Dublin. These facilities are physically located in other molecular biology laboratories, and measures are taken to minimize contamination of ancient individuals, including head-to-toe suits, face masks, hair nets, multiple layers of gloves, bleaching of all surfaces and UV decontamination of all (nonsensitive) reagents. All laboratory tools used to process samples were decontaminated using bleach (1:5 concentration) and UV irradiated in a cross-linker. The final step of library preparation (amplification) was performed outside the ancient DNA laboratory. We included extraction negative controls (no powder), libraries and PCR negative controls (extract was supplemented with water) in every batch of samples processed and carried out them through the entire wet laboratory processing to test for reagent contamination.

Samples were initially UV irradiated on both sides for $\sim 10$ minutes. We targeted the inner ear region of the petrous bone 68,69 using a sandblaster (Renfert). Fragments of the cochlea were then powdered using a mixer mill (Retsch Mixer Mill 400). Twenty-five petrous bone samples were initially screened. Using $75 \mathrm{mg}$ of powder, DNA was extracted using an optimized DNA extraction protocol ${ }^{70}$. Illumina sequencing libraries were constructed using $12.5-25 \mu \mathrm{L}$ of extract and amplified using Accuprime Pfx Supermix (Thermo Fisher Scientific), following Gamba et al. ${ }^{71}$, a protocol adopted from

72. Quality assessment of the amplified library was performed on an Agilent 2100 Bioanalyzer and a Qubit 2.0 Fluorometer. All amplified libraries were initially screened using an Illumina MiSeq. After screening, additional libraries were sequenced to $\sim 1 \mathrm{X}$ on the NextSeq platform.

\section{Bioinformatic Analysis}

Sequencing reads were trimmed using cutadapt (Version. 1.2.1) ${ }^{73}$ and aligned to the human reference genome (GRCh37), with the mitochondrial genome replaced by the revised Cambridge reference sequence (rCRS) using BWA ${ }^{74}$ (Version 0.7.5). Duplicate mapped reads were removed using Picard Tools ${ }^{75}$. Reads with mapping qualities below 30 
were also removed. Unique and filtered reads were analysed with qualimap- ${ }^{76}$ to assess the coverage of the genomes. MapDamage- 277 was used to estimate the level of deamination and the authenticity of the data. We have clipped two bases per read end to minimize the effect of damage.

Pseudo-haplotypes were called using sequenceTools ${ }^{78}$ filtering the calls with mapping and base quality below 30 . As a reference panel we used the positions of the $1240 \mathrm{k}$ capture dataset ${ }^{8}$. The Verteba calls were merged with a panel of 750 modern individuals from 46 populations $^{3}$ and 611 individuals from 67 ancient populations ${ }^{3-5,8,13,37,45,56,57,79-88}$. Molecular sex was determined using ry_compute.py ${ }^{89}$, we also explored the presence of familiar relationshipls with READ ${ }^{90}$.

Mitochondria aligned reads were processed with Schmutzi ${ }^{91}$ to generate a consensus sequence of the mitochondrial genomes using -uselength option. We determined the mitochondrial haplogroups of the mitochondrial consensus sequences with Haplogrep v2.0 ${ }^{92}$. Y chromosome haplogroups of male individuals were determined using Yleaf ${ }^{93}$.

Principal component analysis was built with 597,573 SNPs and 750 modern genomes using smartpca from Eigensoft package ${ }^{46}$. Resulting data was plotted using $\mathrm{R}^{94}$. Ancient samples were projected in the PCA built with the modern ones using the option Isqproject. Two rounds of outlier removal were used. The results were plotted with $\mathrm{R}$.

An unsupervised ADMIXTURE analysis was performed with ADMIXTURE ${ }^{47} .611$ ancient individuals, 2068 modern individuals together with the 20 Verteba individuals were used for the analysis. From the 597,573 SNPs of the Human Origins dataset was filtered removing SNPs with MAF below 0.05 and more than $5 \%$ of missing sites. Filtered SNPs were pruned by linkage-disequilibrium (LD) using PLINK $1.9{ }^{95}$ flag -indep-pairwise with a windows size of 200 SNPS, advanced by 50 SNPs and establishing a $\mathrm{r} 2$ threshold of 0.4 . The ADMIXTURE analysis was performed with 417,913 SNP s, with $\mathrm{K}$ ranging from 2 to 15 and 10 bootstrap replications. Admixture was plotted with $\mathrm{R}$.

F-statistics were run using admixtools ${ }^{46}$ in the form $f_{3}$ (Test, $\mathrm{X}$; Mbuti) using all the ancient European populations available. D statistics were also run using the same package. We have used the form $f_{4}$ (Mbuti, Test; PopA, PopB) using a list of possible sources of Hunter-Gatherer, Neolithic and steppe components. In this analysis, we excluded results with less than 100,000 shared SNPs.

We performed qpAdm using the admixtools package ${ }^{46}$ In this analysis we have used the same proxies as the ones used for D statistic plus Verteba in case of modeling VERT113 and VERT114 individuals and setting allSNPs: NO. The list of samples included in each category is displayed in table S5. As outgroups we used:

Russia_Kostenki14.SG,Italy_North_Villabruna_HG,Han.DG,Mbuti.DG,ONG.SG,Papuan.SDG,Russia_MA1_HG.SG,Ust_Ishim. In a second round we added the rest of tested populations to the right making the models compete between them as described in Harney et ${ }^{96}$. We also performed qpWave analysis to assess the presence of substructure in the Verteba_Trypillia population using the same software and right population set listed below, the threshold was set to a pvalue of 0,05 .

We calculated the ROH segment distribution following the protocol described in Ringbauer et al 2020. ${ }^{48}$. The phenotypic positions analysed were genotyped using pseudohaploid calls. The frequencies of the present-day populations were obtained from the 100 genomes data ${ }^{97}$.

\section{Results}

DNA was extracted from 20 petrous bones. We directly radiocarbon dated 8 of its and determined that six date to approximately 3789-3650 BCE (Late Eneolithic), one individual (VERT-113) from Site 7 dates to 1952-1774 BCE (Early 
Bronze Age, EBA) and one from Site 17 (VERT-114) dates to 980-948 BCE (Late Bronze Age, LBA) (Table S1). The endogenous content of the sequenced samples ranged from 59 to $82 \%$ and yielded genomic coverages between $0.2 \mathrm{X}$ and 2.2X (Table S1). Aligned reads were authenticated using dedicated ancient DNA pipelines (Supplementary methods). All analysed sequences showed the typical pattern of aDNA: an excess of C>T transition in the 5 ' end and G>A transitions in the $3^{\prime}$ end, consistent with the age of the samples; additionally, no signs of contamination were found in the sequences ${ }^{41}$. Further details of the contamination assessment are provided in the Supplementary information. We were able to assign molecular sex to all the individuals, of which 8 were female and 12 were male (Table S1). In all cases, the molecular sex is concordant with morphological sex. No familiar relationships were identified in the analysed data.

\section{Uniparental Markers}

The analysed individuals who are generally thought to come from the Eneolithic Period have maternal haplogroups T2b, H, HV, K1, N1, J1, U5 and T2c (Table S1). The EBA sample shows haplogroup HV, typical from several Neolithic cultures such as the ALPC ${ }^{5}$ as well as European Bronze Age individuals ${ }^{42,43}$. The LBA individual shows haplogroup T2, which is also associated with multiple BA individuals and cultures ${ }^{43-45}$. These haplogroups are typically found in European Neolithic and Bronze Age populations ${ }^{5,13,43}$. Male individuals exhibit Y-chromosome haplogroups G, I and C, which have also been previously reported in Neolithic and Bronze Age populations of Europe ${ }^{13}$. Both the mtDNA and Y-chr haplogroups of all individuals were fully concordant with the previously reported data. (Table S1).

\section{Population Genetics}

To place VC individuals within present-day and archeological Eurasian populations, we used a principal component analysis (PCA) ${ }^{46}$ built with 729 modern individuals from Europe and the Mediterranean Basin ${ }^{3}$. Together with the 20 Verteba genomes, 478 additional ancient genomes (Table S2) were projected onto the PCA. Eighteen out of the $20 \mathrm{VC}$ individuals were placed close to Neolithic and Eneolithic European populations, such as LBK, Central European Middle and Late Neolithic samples and Moldova Trypillian individuals ${ }^{37}$ (Fig. 2A). The PCA also evidenced the extreme similarity between the 18 newly reported Trypillians and the other 4 Trypillians from Verteba cave previously sequenced $^{13}$; therefore, all 22 individuals were labeled together as Verteba_Trypillia and further analysed together. The two Bronze Age individuals are clear outliers. Individual VERT-114 falls within the Bell-Beaker diversity and appears to have a position close to the Czech, Hungarian and Polish Bell-Beaker groups. Individual VERT-113 appears close to European Corded-Ware and Srubnaya populations, showing a strong affinity to steppe samples. We then explored the presence of structure in the Trypillian population (using only the 22 samples from the main cluster) using qpWave. The results showed the absence of population structure; therefore, all samples were analysed together, as no individual showed statistically significant pairwise differences from the rest using a threshold of 0.05 (Fig. 2C).

We then explored the genetic diversity of VC individuals using ADMIXTURE ${ }^{47}$. The 22 individuals (labeled 'Verteba_Trypillia') in the PCA that showed affinity with Eneolithic samples are mostly defined by the ancestral component dominant in Anatolia-Neolithic individuals, which suggests a strong relationship with European Neolithic populations, similar to previous studies ${ }^{13,37}$. However, these samples also show the presence of EHG, CHG, and WHG components as described in Mathiesson et al. ${ }^{13}$, with the exception of one individual (I3151), who seems to be absent of any EHG/CHG ancestry. Individual VERT-114 (LBA) shows a predominant Anatolia Neolithic component and a great presence of an EHG component. The EBA individual (VERT-113) exhibits a high degree of similarity with the Corded Ware and Yamnaya steppe populations (Fig. 2B, Fig. S1). 
Next, we investigated the genetic affinities of the VC individuals using $f$-statistics. We used $f_{3}$-outgroup statistics to quantify the amount of shared genetic drift of Verteba_Trypillia, VERT114, and VERT113, tested against other ancient European populations. Overall, the Verteba_Trypillia individuals share more derived SNPs with Neolithic European populations (Fig. 3). Individual VERT-114 shows a high level of derived SNPs with HG populations as well as with Late Neolithic and Bronze Age populations. In turn, individual VERT-113 shares derived SNPs with HG populations and some steppe-related populations, such as the Central European Corded Ware.

We ran $f_{4}$ statistics and qpAdm ${ }^{46}$ to quantify ancestry components as well as to estimate the direction of gene flow (Table S3, Fig. S2). We ran several tests to understand the genetic composition of Verteba trypsia (excluding the outliers) and the possible sources of genetic admixture for this population. We first ran qpAdm using populations chronologically close to the CTCC individuals. Five models worked (Table S4), with the simplest ones involving approximately $93 \%$ of Hungary_LateC_EBA_Baden_Yamnaya ancestry plus 7\% from Yamnaya-related populations, evidencing the connection between Trypillians and steppe populations, as Hungary_LateC_EBA_Baden_Yamnaya also has Steppe ancestry (Fig. 4). We then tested possible connections with specific steppe-related populations using the $f_{4}$ statistical test in the form $f_{4}$ (Mbuti,Verteba_Trypillia;Russia_Samara_EBA_Yamnaya,Ukraine_EBA_Yamnaya) $=-0.000525$, Z score $=-1.843$, which does not statistically connect the Trypillians with the Ukrainian or Russian Yamnaya populations.

We then explored the same individuals, but this time using populations representing different basal ancestries that might have contributed to the CTCC genetic pool (EHG, CHG, Anatolia_N, WHG and Ukranie_N) (Table S5). Only one model worked, which involved 40\% Anatolia Neolithic-related ancestry, 20\% WHG and 40\% CHG. These results were also observed in Mathiesson et al 2018 with very similar proportions but were not feasible in the Moldovan Trypillians from Immel et al. (2020). To investigate the possible sources of the HG component of the 22 Verteba_Trypillia individuals, we ran statistics in the form of $f_{4}$ (Mbuti, Verteba_Trypillia;Ukraine_N,Serbia_IronGates_Mesolithic) $=0.001603, Z$ score= $12.173, f_{4}$ (Mbuti, Verteba_Trypillia;Ukraine_N,Loschbour) $=0.001730, Z$ score $=6.380, f_{4}$ (Mbuti, Verteba_Trypillia;Ukraine_N,Koros-HG) $=0.002037$, Z score $=7.573, f_{4}$ (Mbuti, Verteba_Trypillia;KorosHG,Serbia_IronGates_Mesolithic) $=-0.00047$, Z score $=-1.879$. This result shows that Koros-HG is the WHG source with the highest genetic affinity to Ukraine Trypillians. When compared to Central European Eneolithic populations, the Eneolithic Verteba individuals do not seem to share a statistically significant affinity with the CTCC population of Moldova, as shown in the form of $f_{4}$ (Mbuti, Verteba_Trypillia;Hungary_LateC_EBA_Baden_Yamnaya, Trypillia_Moldova):, 0.000304 Z score $=-0.936$. qpAdm also indicates that Ukraine_N and WHG are two likely sources of HG-related ancestry to Verteba_Trypillia, in addition to Hungary_LateC_EBA_Baden_Yamnaya (Table S4). The statistic $f_{4}($ Mbuti, Verteba_Trypillia; Ukraine_N, WHG) shows a clear tendency towards WHG: 0.001192, Z score=6.26, suggesting very little presence of ancestry from the local Hunther_Gatherers in the Verteba individuals.

To detect different individual ancestry compositions, we ran qpAdm tests individually on the 22 Verteba_Trypillian individuals, showing that most of them can be modeled with a single source using Trypillia_Moldova, Hungary_LateC_EBA_Baden_Yamnaya or Hungary_LN_Tisza, indicating a clear affinity for Late_Neolithic populations with steppe ancestry (Table S6), as all these populations show the presence of a steppe component. Surprisingly, only four of the Verteba_Trypillia individuals can be modeled using Trypillia-Moldova as a single source. To investigate whether there were statistically significant differences between the possible sources for these individuals, we ran tests $f_{4}$ (Mbuti, VERTXXX; Hungary_LateC_EBA_Baden_Yamnaya, Trypillia-Moldova) individually (Table S7). The results show that only one individual (VERT035) is statistically more related to Hungary_LateC_EBA_Baden_Yamnaya than to Trypillian-Moldova, pointing towards the existence of some variability within the Trypillians in Ukraine. As we did for the general population, we also performed qpAdm analyses using distal sources (EHG, CHG, Anatolia_N, WHG and Ukranie_N). The results show that most of the individuals can be modeled using Anatolia_N plus 20\% WHG/Ukranie_N. When $f_{4}$ (Mbuti, VERTXXX; Ukraine_N, WHG) was tested individually, the statistics showed no differences (Table S8); 
however, the affinity to WHG at the population level was clear, revealing the importance of having large sample sizes to perform f-statistics-based assessments (Table S3).

VERT-113, dated to around the EBA, shows a clear signal of steppe-related ancestry and is the only individual in the dataset that shows a strong influx of this ancestry: $f_{4}$ (Mbuti, Verteba_EBA; Russia_Samara_EBA_Yamnaya, LBK): $-0.00398 \mathrm{Z}$ score=-7.848. The same test with individual VERT-114 was not statistically significant $(Z=1.382)$. Relevantly, we observe a major affinity to Russia_Yamnaya over Ukraine_Yamnaya using $f_{4}$ statistics (Table S4). Furthermore, this is the only individual who shows a major affinity to Ukranian_N over WHG as the source of HG-related ancestry, as shown by the statistic $f_{4}($ Mbuti, VERT-113; Ukraine_N, WHG): -0.001276 , Z score (Z=-4.202). The distal models of qpAdm using basal ancestries reveal that this individual exhibits up to $33 \%$ Ukraine_N and 66\% CHG, supporting high amounts of steppe-related ancestry. When modeled with close chronology populations, the individual requires a single source related to the Corded_Ware (Fig. 4, Table S4). We tried to assess whether the signal could, however, correspond to similar genetic populations but more contemporary and geographically closer to VERT-113, such as Srubnaya, using the statistic $f_{4}$ (Mbuti, VERT-113; Poland_Southeast_CordedWare, Russia_Srubnaya), but the results $\left(f_{4}=0.00003, Z=0.11\right)$ show that there is no statistical relationship, which indicates no evidence supporting the Srubnaya origin.

Individual VERT-114, dated around the LBA, showed a genetic position close to Bell-Beaker populations in PCA and ADMIXTURE. This individual shows a higher influx of ancestry from WHG than from EHG populations $f_{4}$ (Mbuti, VERT114; WHG, EHG) -0.002, Z score=-8,64), similar to the results obtained for the aggregate group of 22 Verteba individuals. qpAdm results for this individual show that a single model with a Bell_Beaker population as a single source works (Fig. 4). Many of the two-way models involve populations related to Ukraine_Globular_Amphora and to steppe populations, with approximately $60 \%$ of ancestry from the former and the rest from the latter. We also explored the possible connections between this individual and the Cimmerians, who established themselves in present-day Ukraine around the year $1000 \mathrm{BC}$, being contemporary to VERT-114. Both $f_{4}$ (Mbuti, VERT-114; Moldova_Cimmerian. SG, Poland_Southeast_BellBeaker) $f_{4}=0.00428$ Zscore=7.171) and $f_{4}$ (Mbuti, VERT-114; Moldova_Cimmerian. SG, Hungary_EBA_BellBeaker) $f_{4}=0.003301$, Zscore $=4.043$ ) showed a clear affinity of VERT-114 to the Bell_Beaker individuals over the Cimmerians. This individual can also be modeled as an admixture between Turkey_N (67\%) and Ukraine_N (33\%) (Table S5).

We used the approach presented in Ringbauer et al $2020^{48}$ to explore the presence of runs of homozygosity (ROH) in the sample. We observed that the samples tested presented very few parts of the genome under ROH (Fig. S3), meaning that the individuals were part of large populations. An exception was VERT-100, who showed long ROH segments, suggesting that this individual was an offspring of related individuals.

\section{Phenotypic Positions}

We genotyped 105 SNPs linked with metabolic, pigmentation and pathogen resistance phenotypic traits. Pseudohaploid genotypes are shown in Table S9. From these genotypes, it is evident that none of the tested individuals from Verteba cave were lactose tolerant, as all are homozygous for the nontolerant variants for SNPs rs $4988235^{49}$ and rs $182549^{50}$. It is also interesting to remark that, except for two individuals, the majority of individuals from Verteba cave have the variant of SNP rs12913832 associated with blue eyes and the other two associated with dark eyes ${ }^{51}$.

\section{Discussion}

The CTCC is an important archaeological complex that brought farming to Eastern Europe ${ }^{16,18}$. Prior to our research, four individuals from Verteba Cave and four individuals from Moldova were analysed using nuclear DNA; nevertheless, the 
previously reported diversity within the $\mathrm{CTCC}^{37}$ showed that more research on the genetic diversity of this culture is needed to understand its origin, dynamics, and collapse. Recent publications have revealed the utility and relevance of large-scale projects focused on specific sites ${ }^{43,52}$. Here, we have presented genomic data from 20 individuals buried in Verteba Cave that are dated to the 4th, 2nd and 1st millennia BC. The genetic analysis of these individuals has revealed important genetic turnover both in the Early Bronze Age and during the Late Bronze Age. In the future, more individuals should be sequenced to clarify these observations, in particular to obtain more individuals from the third millennium BCE onwards, as the genomic record in Ukraine from the Bronze Age is limited to six individuals from the 2nd and 3rd millennia BC. Importantly, we also provide eight new radiocarbon dates, which are extremely relevant, as previous studies have demonstrated the disturbed nature of much of Verteba Cave's stratigraphy, caused by repeated use of the site from at least the Mesolithic up until modern times. ${ }^{28}$

Previous analyses of CTCC individuals' mitochondrial DNA HVRI indicated their close maternal ancestry with early Neolithic groups, with lineages that are representative of the Neolithic 'package', including haplogroups $H, H V, T, V, J$, and $\mathrm{K}^{53,54}$. With the exception of two individuals with haplogroup U5a, all the other 18 individuals who were included in our analyses have haplogroups that are similar to Central European Neolithic groups ${ }^{13}$. This diversity is in stark contrast to individuals from earlier non-agricultural Neolithic sites from the Ukraine that have only haplogroup $U$, likely the result of continuity with previous Mesolithic hunter-gatherers (HG). The mtDNA haplogroup diversity suggests that local populations were largely replaced by those associated with the Trypillian culture. The majority of VC individuals exhibit the G2a2 Y haplogroup, which is widely present in Anatolia-related Neolithic European individuals ${ }^{5,13}$. The other identified haplogroups, C1 and 12, have also been reported among European Neolithic populations, pointing to an origin of the CTCC individuals without a sex-biased migratory past, which contrasts with the steppe migrations during the Bronze Age ${ }^{55}$.

Population genetic analyses indicate that the individuals buried at Verteba Cave during the Late Eneolithic (3789-3650 BCE) genetically resemble other previously published CTCC individuals and are closely related to other published CTCC individuals in Moldova ${ }^{37}$. These observations broadly suggest that Eneolithic CTCC descended from the same, or closely related, population that spread the Neolithic across most of Europe and without little or no sign of admixture with earlier Ukrainian Mesolithic or Neolithic groups composed of hunter-gatherer-related ancestry and specifically pointing towards the Baden individuals from Hungary. In fact, most of the Trypillian individuals can be modeled by Eneolithic populations from Europe that have steppe ancestry; however, 4 out of the 20 individuals could be modeled as Moldovan Trypillians. These results in the qpAdm modeling suggest that there were differences in the ancestry composition of the Trypillians of Verteba cave, which could be linked to the proportion of HG in the individuals, although this variability is not substantial enough to differentiate the individuals into different populations.

Previous studies of CTCC individuals could not provide a clear origin for the HG component of CTCC-associated groups. Here, although models including Ukranian_N individuals and WHG seem to work, the $f_{4}$-statistics suggest that the source of the HG component would be mainly WHG. In addition, not a single qpAdm model using EHG as a source works, which supports that observation. The significant proportion of WHG ancestry found in the Trypillians (up to 18\%) might be related to the hunter gatherer resurgence seen in other Middle Neolithic populations of Central Europe, likely due to admixture with groups in the west who already had a higher WHG component derived from Anatolia-related Neolithic groups prior to the origin of the CTCC $8,13,56$. This would also indicate that the HG Neolithic populations from Ukraine did not contribute much ancestry to the Trypillians. In addition, we also observed the presence of steppe-related ancestry in these individuals, as was revealed in Moldova ${ }^{37}$, although the proportion in the Verteba individuals was lower, which could correlate with the age of the individuals, suggesting a continuous pulse from the East to the West gradually increasing the Yamnaya-related ancestry during the 4th millennium. 
Individual VERT-113, dated to the EBA (1952-1774 BCE), has an ancestry profile that is quite different from the earlier CTCC individuals. There was significantly more Caucasus HG/Yamnaya and EHG ancestry, and thus, this individual was related to Yamnaya expansions. qpAdm results suggest a link between VERT-113 and Corded Ware populations from Poland, pointing to a similarity between this individual and these populations. Additionally, this is the only individual with a higher genetic affinity to Ukraine_N than to WHG, suggesting that the population that originated EBA in the 2nd millennium BC may have shared affınities with the Ukraine_N populations.

Interestingly, VERT-114 (Late Bronze Age) does not show many genetic connections with EBA VERT-113 according to the $f_{3}$ values, which is clearly associated with Yamnaya pastoralists. The genomic composition of VERT-114 suggests a relationship with Beaker-related populations, despite being almost 1,000 years younger than the end of the Bell Beaker phenomenon ${ }^{57}$, and with a date that would be more coincident with the Cimmerians or Scythians ${ }^{58}$. However, no qpAdm models with these cultures work, and the $f_{4}$ results seem to confirm the similarity with the Bell Beakers over the Cimmerians. The genetic background of this individual, with its strong Western affinities, supports the evidence shown in Narasimhan et al. ${ }^{59}$ of a western influx into the steppe during the Late Bronze Age.

Our radiocarbon dates show that the cave was used during at least three different periods (CTCC, Bell-Beaker, and a third group that could be either Corded Ware or Yamnaya-related pastoralists). Further sequencing and analysis of individuals from the cave and the area surrounding VC dating from the third millennium BCE will be critical for exploring cave use after abandonment by CTCC-related people.

In conclusion, the results show that Verteba Cave represents a significant mortuary site that connects East and West. The genetic structure of the CTCC peoples includes ancestry related to both earlier hunter gatherers from the west and farmers from the Near East and one that is genetically distinct from those of Moldovan CTCC peoples. The lack of local ancestry associated with Ukrainian Neolithic hunter gatherers suggests that these farmers mostly replaced local foragers and did not mix with the neighbouring steppe populations. Additionally, during the Bronze Age, Verteba Cave was used by successive waves of nomadic pastoralists from the east that eventually brought significant genetic and cultural changes to Europe that eventually mixed with the local Trypillians. Additional genomic sampling from these later time periods will help to answer questions of site chronology and possibly indicate how the Trypliians eventually collapsed.

\section{Declarations}

\section{Author Contributions}

RWS and JKK collected samples, RWS performed wet laboratory work, PG and

DMF analysed the data, TKH and DJK performed radiocarbon dating and isotope

analysis, PG, RWS, DMF, JKK, SHL, TKH, HO and RP wrote the paper with input from all coauthors.

Funding was provided by the European Commission's Marie Skłodowska-Curie

Individual Fellowships (PACE \#70245 to RWS and RP), Grand Valley State

University Professional Development Grant (GDM), the University of

Wisconsin-Oshkosh (JKK) and the National Science Foundation (BCS-1725067 to TKH and DJK).

Sequencing reads have been deposited in the European Nucleotide Archive (ENA) with the accession code XXX.

We declare no conflict of interest.

Page $11 / 20$ 


\section{References}

1. Cavalli-Sforza, L. L. Genes, peoples, and languages. Proc. Natl. Acad. Sci. U. S. A. 94, 7719-7724 (1997).

2. Shennan, S. The First Farmers of Europe: An Evolutionary Perspective. (Cambridge University Press, 2018).

3. Lazaridis, I. et al. Genomic insights into the origin of farming in the ancient Near East. Nature 536, 419-424 (2016).

4. Hofmanová, Z. et al. Early farmers from across Europe directly descended from Neolithic Aegeans. Proc. Natl. Acad. Sci. U. S. A. 113, 6886-6891 (2016).

5. Lipson, M. et al. Parallel palaeogenomic transects reveal complex genetic history of early European farmers. Nature 551, 368-372 (2017).

6. Ancient Europe 8000 B.C. - A.D. 1000 - (Encyclopedia of the Barbarian World - Vol. 1. (Charles Scribner \& Sons, 2004).

7. Barton, L. First Farmers: The Origins of Agricultural Societies by P. S. Bellwood, and: The Peopling of East Asia: Putting Together Archaeology, Linguistics and Genetics ed. by L. Sagart, R. Blench, and A. Sanchez-Mazas, and: The Origins of Pottery and Agriculture ed. by Y. Yasuda (review). Asian Perspectives 51, 321-333 (2012).

8. Haak, W. et al. Massive migration from the steppe was a source for Indo-European languages in Europe. Nature 522, 207-211 (2015).

9. Skoglund, P. et al. Origins and genetic legacy of Neolithic farmers and hunter-gatherers in Europe. Science 336, 466469 (2012).

10. Zvelebil, M. \& Dolukhanov, P. The transition to farming in Eastern and Northern Europe. Journal of World Prehistory $\mathbf{5}$, 233-278 (1991).

11. Zvelebil, M. \& Rowley-Conwy, P. Transition to farming in Northern Europe: A hunter-gatherer perspective. Norwegian Archaeological Review 17, 104-128 (1984).

12. Jones, E. R. et al. The Neolithic Transition in the Baltic Was Not Driven by Admixture with Early European Farmers. Curr. Biol. 27, 576-582 (2017).

13. Mathieson, I. et al. The genomic history of southeastern Europe. Nature 555, 197-203 (2018).

14. Ellis, L. The Cucuteni-Tripolye Culture: a study in technology and the origins of complex society. vol. 217 (BAR Oxford, 1984).

15. Milisauskas, S. \& Kruk, J. Middle Neolithic/Early Copper Age, Continuity, Diversity, and Greater Complexity, 5500/5000-3500 BC. in European Prehistory: A Survey (ed. Milisauskas, S.) 223-291 (Springer New York, 2011).

16. Zbenovich, V. G. The Tripolye culture: Centenary of research. Journal of World Prehistory 10, 199-241 (1996).

17. Rassamakin, Y., Menotti, F. \& Korvin-Piotrovskiy, A. Absolute chronology of Ukrainian Tripolye settlements. The Tripolye Culture Giant-Settlements in Ukraine. Formation, Development and Decline. Oxford: Oxbow Books 19-69 (2012).

18. Menotti, F. \& Korvin-Piotrovskiy, A. The Tripolye culture giant-settlements in Ukraine : formation, development and decline. 264 S. (Oxbow Books, 2012).

19. Marinescu-Bîlcu, S. Cultura Precucuteni pe teritoriul României, Bucureşti: Ed. Academiei RSR (1974). 
20. Rassmann, K. et al. High precision Tripolye settlement plans, demographic estimations and settlement organization. Journal of Neolithic Archaeology 96-134 (2014).

21. Harper, T. K., Diachenko, A., Rassamakin, Y. Y. \& Kennett, D. J. Ecological dimensions of population dynamics and subsistence in Neo-Eneolithic Eastern Europe. Journal of Anthropological Archaeology 53, 92-101 (2019).

22. Дергачев, В. А. О скипетрах, о лошадях, о войне. (Нестор-История, 2007).

23. Videiko, M. Y. Reasons of origins and development of the Tripolian proto-cities. Archaeology 4, 145-151 (1998).

24. Diachenko, A. \& Menotti, F. The gravity model: monitoring the formation and development of the Tripolye culture giant-settlements in Ukraine. J. Archaeol. Sci. 39, 2810-2817 (2012).

25. Müller, J., Rassmann, K. \& Videiko, M. Trypillia Mega-Sites and European Prehistory: 4100-3400 BCE. (Routledge, 2016).

26. Круц, В. А. Позднетрипольские памятники Среднего Поднепровья. (Наук. думка, 1977).

27. Великанова, М. С. Палеоантропология Прутско-Днестровского междуречья. (наука, 1975).

28. Lillie, M. C. et al. First isotope analysis and new radiocarbon dating of Trypillia (Tripolye) farmers from Verteba Cave, Bilche Zolote, Ukraine. Documenta Praehistorica 44, 306 (2017).

29. Madden, G. D., Karsten, J. K., Ledogar, S. H., Schmidt, R. \& Sokhatsky, M. P. Violence at Verteba Cave, Ukraine: New insights into the Late Neolithic intergroup conflict. Int. J. Osteoarchaeol. 28, 44-53 (2018).

30. Nikitin, A. G., Sokhatsky, M. P., Kovaliukh, M. M. \& Videiko, M. Y. Comprehensive site chronology and ancient mitochondrial DNA analysis from verteba cave-a trypillian culture site of eneolithic Ukraine. Interdiscip. Archaeol. 1, 918 (2010).

31. Ledogar, S. H. et al. New AMS Dates for Verteba Cave and Stable Isotope Evidence of Human Diet in The Holocene Forest-Steppe, Ukraine. Radiocarbon 61, 141-158 (2019).

32. Nikitin, A. g., Sokhatsky, M. p., Kovaliukh, M. M. \& Videiko, M. y. Comprehensive site chronology and ancient Mitochondrial dna analysis from Verteba cave - a trypillian culture site of eneolithic ukraine. Plos One (2017).

33. Kadrow, S. \& Pokutta, D. A. The Verteba cave: a subterranean sanctuary of the Cucuteni-Trypillia culture in Western Ukraine. Journal of Neolithic Archaeology (2016).

34. Ledogar, S. H. A zooarchaeological and geochemical analysis of the faunal remains from the Tripolye site Verteba Cave, Ukraine. (State University of New York at Albany, 2017).

35. Anthony, D. W. The Horse, the Wheel, and Language: How Bronze-Age Riders from the Eurasian Steppes Shaped the Modern World. (Princeton University Press, 2007).

36. Schmidt, R. W. et al. Analysis of ancient human mitochondrial DNA from Verteba Cave, Ukraine: insights into the Late Neolithic-Chalcolithic Cucuteni-Tripolye culture. Anthropol. Sci. advpub, (2020).

37. Immel, A. et al. Gene-flow from steppe individuals into Cucuteni-Trypillia associated populations indicates longstanding contacts and gradual admixture. Sci. Rep. 10, 4253 (2020). 
38. Ellis, L. Analysis of Cucuteni-Tripolye and Kurgan Pottery and the Implications for Ceramic Technology in The Transformation of European and Anatolian Culture 4500-2500 BC and Its Legacy. Journal (The) of Indo-European Studies Washington, DC 8, 211-230 (1980).

39. Tsvek, E. \& Rassamakin, Y. Y. The interactions between the Eastern Tripolye culture and the Pontic steppe area: Some aspects of the problem. Cucuteni 120, 173-192 (2005).

40. Chapman, J. Expansion and social change at the time of Varna. Counterpoint: essays in Archaeology and Heritage Studies in honour of Professor Kristian Kristiansen 301-308 (2013).

41. Sawyer, S., Krause, J., Guschanski, K., Savolainen, V. \& Pääbo, S. Temporal patterns of nucleotide misincorporations and DNA fragmentation in ancient DNA. PLoS One 7, e34131 (2012).

42. Olalde, I. et al. The genomic history of the Iberian Peninsula over the past 8000 years. Science $363,1230-1234$ (2019).

43. Mittnik, A. et al. Kinship-based social inequality in Bronze Age Europe. Science 366, 731-734 (2019).

44. Saag, L. et al. The Arrival of Siberian Ancestry Connecting the Eastern Baltic to Uralic Speakers further East. Curr. Biol. 29, 1701-1711.e16 (2019).

45. Wang, C.-C. et al. Ancient human genome-wide data from a 3000-year interval in the Caucasus corresponds with ecogeographic regions. Nat. Commun. 10, 590 (2019).

46. Patterson, N. et al. Ancient admixture in human history. Genetics 192, 1065-1093 (2012).

47. Alexander, D. H., Novembre, J. \& Lange, K. Fast model-based estimation of ancestry in unrelated individuals. Genome Res. 19, 1655-1664 (2009).

48. Ringbauer, H., Novembre, J. \& Steinrücken, M. Detecting runs of homozygosity from low-coverage ancient DNA. bioRxiv 2020.05.31.126912 (2020) doi:10.1101/2020.05.31.126912.

49. Enattah, N. S. et al. Identification of a variant associated with adult-type hypolactasia. Nat. Genet. 30, 233-237 (2002).

50. Bersaglieri, T. et al. Genetic signatures of strong recent positive selection at the lactase gene. Am. J. Hum. Genet. 74, 1111-1120 (2004).

51. Eiberg, H. et al. Blue eye color in humans may be caused by a perfectly associated founder mutation in a regulatory element located within the HERC2 gene inhibiting OCA2 expression. Hum. Genet. 123, 177-187 (2008).

52. Amorim, C. E. G. et al. Understanding 6th-century barbarian social organization and migration through paleogenomics. Nat. Commun. 9, 3547 (2018).

53. Brandt, G. et al. Ancient DNA reveals key stages in the formation of central European mitochondrial genetic diversity. Science 342, 257-261 (2013).

54. Nikitin, A. G. et al. Mitochondrial DNA analysis of eneolithic trypillians from Ukraine reveals neolithic farming genetic roots. PLoS One 12, e0172952 (2017). 
55. Goldberg, A., Günther, T., Rosenberg, N. A. \& Jakobsson, M. Ancient X chromosomes reveal contrasting sex bias in Neolithic and Bronze Age Eurasian migrations. Proc. Natl. Acad. Sci. U. S. A. 114, 2657-2662 (2017).

56. Mathieson, I. et al. Genome-wide patterns of selection in 230 ancient Eurasians. Nature 528, 499-503 (2015).

57. Olalde, I. et al. The Beaker phenomenon and the genomic transformation of northwest Europe. Nature 555, 190-196 (2018).

58. MacKenzie, D. \& Curran, M. W. A history of Russia, the Soviet Union, and beyond. (Wadsworth Publishing Company, 2002).

59. Narasimhan, V. M. et al. The formation of human populations in South and Central Asia. Science 365, (2019).

60. Kennett, D. J. et al. Archaeogenomic evidence reveals prehistoric matrilineal dynasty. Nat. Commun. 8, 14115 (2017).

61. Lohse, J. C., Madsen, D. B., Culleton, B. J. \& Kennett, D. J. Isotope paleoecology of episodic mid-to-late Holocene bison population expansions in the Southern Plains, U.S.A. Quarternary Science Reviews 102, 14-26 (2014).

62. McClure, S. B., Puchol, O. G. \& Culleton, B. J. Ams dating of human bone from cova de la pastora: New evidence of ritual continuity in the prehistory of Eastern Spain. Radiocarbon 52, 25-32 (2010).

63. van Klinken, G. J. Bone Collagen Quality Indicators for Palaeodietary and Radiocarbon Measurements. J. Archaeol. Sci. 26, 687-695 (1999).

64. Santos, G. M., Southon, J. R., Druffel-Rodriguez, K. C., Griffin, S. \& Mazon, M. Magnesium Perchlorate as an Alternative Water Trap in AMS Graphite Sample Preparation: A Report On Sample Preparation at Kccams at the University of California, Irvine. Radiocarbon 46, 165-173 (2004).

65. Stuiver, M. \& Polach, H. A. Discussion Reporting of 14C Data. Radiocarbon 19, 355-363 (1977).

66. Reimer, P. J. et al. IntCal13 and Marine13 Radiocarbon Age Calibration Curves 0-50,000 Years cal BP. Radiocarbon 55, 1869-1887 (2013).

67. Ramsey, C. B. Bayesian Analysis of Radiocarbon Dates. Radiocarbon 51, 337-360 (2009).

68. Pinhasi, R., Fernandes, D. M., Sirak, K. \& Cheronet, O. Isolating the human cochlea to generate bone powder for ancient DNA analysis. Nat. Protoc. 14, 1194-1205 (2019).

69. Pinhasi, R. et al. Optimal Ancient DNA Yields from the Inner Ear Part of the Human Petrous Bone. PLoS One 10, e0129102 (2015).

70. Dabney, J. et al. Complete mitochondrial genome sequence of a Middle Pleistocene cave bear reconstructed from ultrashort DNA fragments. Proc. Natl. Acad. Sci. U. S. A. 110, 15758-15763 (2013).

71. Gamba, C. et al. Genome flux and stasis in a five millennium transect of European prehistory. Nat. Commun. 5, 5257 (2014).

72. Meyer, M. \& Kircher, M. Illumina sequencing library preparation for highly multiplexed target capture and sequencing. Cold Spring Harb. Protoc. 2010, db.prot5448 (2010).

73. Martin, M. Cutadapt removes adapter sequences from high-throughput sequencing reads. EMBnet.journa/ 17, 10-12 (2011). 
74. Li, H. \& Durbin, R. Fast and accurate short read alignment with Burrows-Wheeler transform. Bioinformatics 25, 17541760 (2009).

75. Picard-tools. http://broadinstitute.github.io/picard.

76. Okonechnikov, K., Conesa, A. \& García-Alcalde, F. Qualimap 2: advanced multi-sample quality control for highthroughput sequencing data. Bioinformatics 32, 292-294 (2016).

77. Jónsson, H., Ginolhac, A., Schubert, M., Johnson, P. L. F. \& Orlando, L. mapDamage2.0: fast approximate Bayesian estimates of ancient DNA damage parameters. Bioinformatics 29, 1682-1684 (2013).

78. Schiffels, S. et al. Iron Age and Anglo-Saxon genomes from East England reveal British migration history. Nat. Commun. 7, 10408 (2016).

79. Fernandes, D. M. et al. A genomic Neolithic time transect of hunter-farmer admixture in central Poland. Sci. Rep. 8, 14879 (2018).

80. Sikora, M. et al. The population history of northeastern Siberia since the Pleistocene. Nature 570, 182-188 (2019).

81. Kılınç, G. M. et al. The Demographic Development of the First Farmers in Anatolia. Curr. Biol. 26, 2659-2666 (2016).

82. Fu, Q. et al. The genetic history of Ice Age Europe. Nature 534, 200-205 (2016).

83. Damgaard, P. de B. et al. 137 ancient human genomes from across the Eurasian steppes. Nature 557, 369-374 (2018).

84. Lazaridis, I. et al. Genetic origins of the Minoans and Mycenaeans. Nature 548, 214-218 (2017).

85. Raghavan, M. et al. The genetic prehistory of the New World Arctic. Science 345, 1255832 (2014).

86. Olalde, I. et al. Derived immune and ancestral pigmentation alleles in a 7,000-year-old Mesolithic European. Nature 507, 225-228 (2014).

87. Lazaridis, I. et al. Ancient human genomes suggest three ancestral populations for present-day Europeans. Nature 513, 409-413 (2014).

88. Keller, A. et al. New insights into the Tyrolean Iceman's origin and phenotype as inferred by whole-genome sequencing. Nat. Commun. 3, 698 (2012).

89. Skoglund, P., Storå, J., Götherström, A. \& Jakobsson, M. Accurate sex identification of ancient human remains using DNA shotgun sequencing. J. Archaeol. Sci. 40, 4477-4482 (2013).

90. Monroy Kuhn, J. M., Jakobsson, M. \& Günther, T. Estimating genetic kin relationships in prehistoric populations. PLoS One 13, e0195491 (2018).

91. Renaud, G., Slon, V., Duggan, A. T. \& Kelso, J. Schmutzi: estimation of contamination and endogenous mitochondrial consensus calling for ancient DNA. Genome Biol. 16, 224 (2015).

92. Weissensteiner, H. et al. HaploGrep 2: mitochondrial haplogroup classification in the era of high-throughput sequencing. Nucleic Acids Res. 44, W58-63 (2016). 
93. Ralf, A., Montiel González, D., Zhong, K. \& Kayser, M. Yleaf: Software for Human Y-Chromosomal Haplogroup Inference from Next-Generation Sequencing Data. Mol. Biol. Evol. 35, 1291-1294 (2018).

94. R Core Team. R: A language and environment for statistical computing. R Foundation for Statistical Computing, Vienna, Austria. (2013).

95. Purcell, S. et al. PLINK: a tool set for whole-genome association and population-based linkage analyses. Am. J. Hum. Genet. 81, 559-575 (2007).

96. Harney, É., Patterson, N., Reich, D. \& Wakeley, J. Assessing the performance of qpAdm: a statistical tool for studying population admixture. Genetics 217, (2021).

97. 1000 Genomes Project Consortium et al. A global reference for human genetic variation. Nature 526, 68-74 (2015).

\section{Figures}
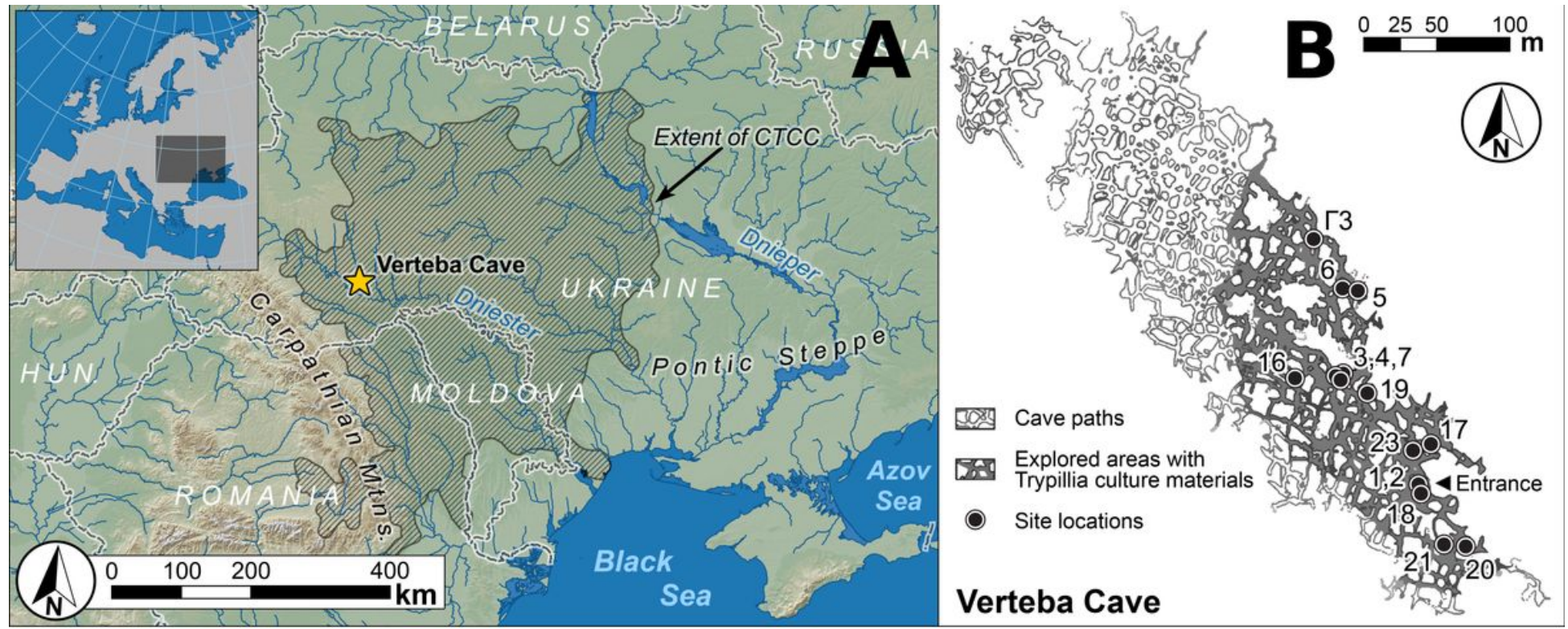

\section{Figure 1}

Verteba Cave: A) Location of Verteba cave in Ternopil Oblasts, Ukraine, plotted against the overall distribution of CTCC sites. B) Map of sites within Verteba cave; individuals included in this publication were found at sites 7, 17 and 20, adapted from Ledogar et al 201931. 


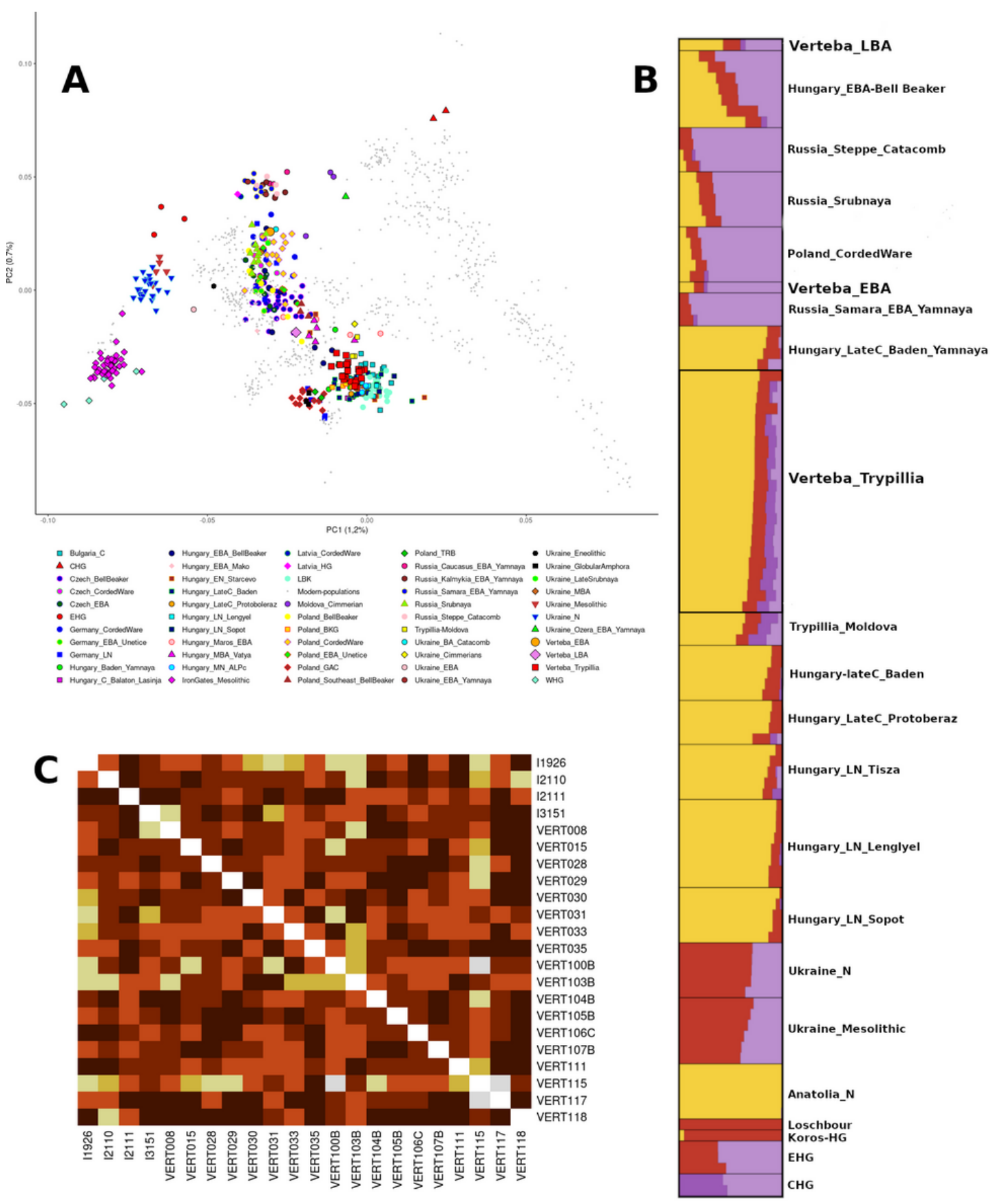

\section{Figure 2}

Population genetics: A) PCA of Neolithic and Bronze Age populations of Eastern Europe. B) Admixture analysis of the most representative populations. C) qpWave plot of the Verteba_Trypillia individuals, where no individuals show a sign to be clustered in a different population. 

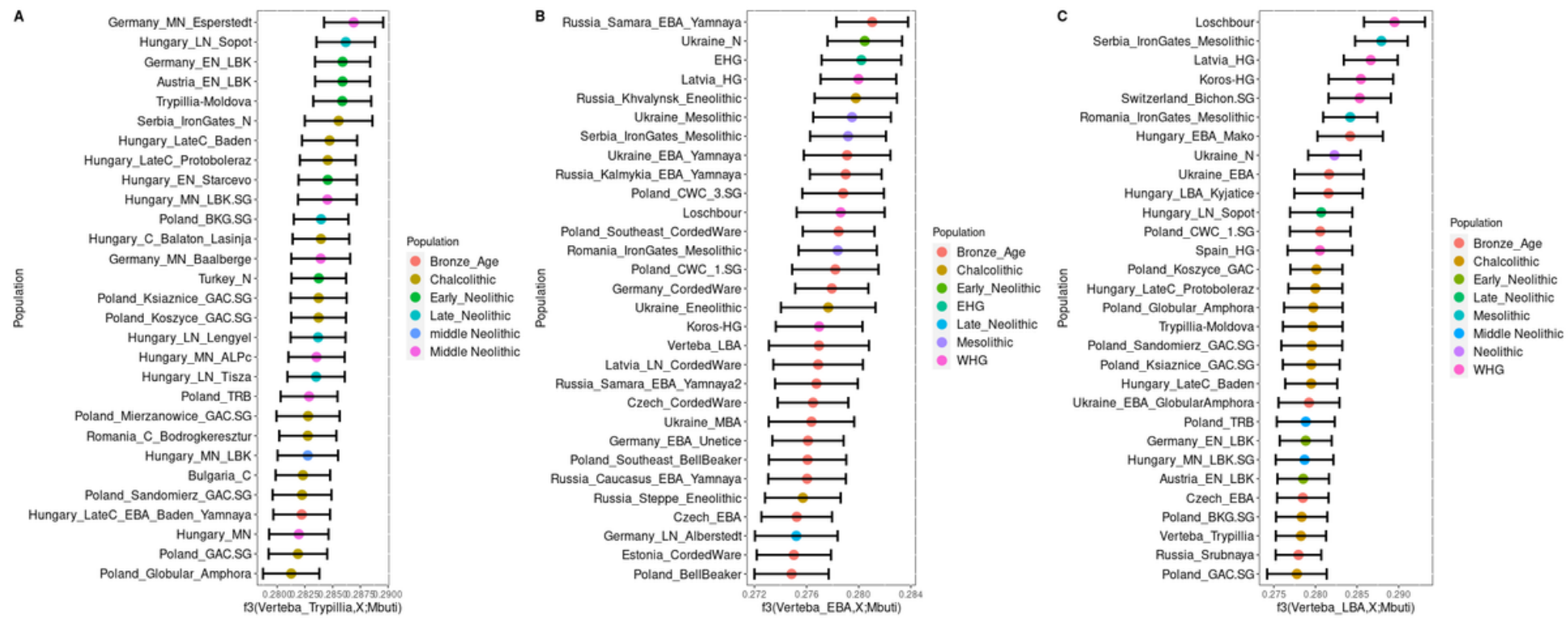

\section{Figure 3}

Outgroup-f3 statistics. The samples were clustered between Verteba individuals, individual VERT114 (Verteba_LBA) and individual VERT113 (Verteba_EBA). We plotted the 20 populations with the highest values.

\begin{tabular}{|c|c|c|c|}
\hline Period & Culture & \multicolumn{2}{|l|}{ Ancestry composition of the individuals } \\
\hline \multirow{2}{*}{$\begin{array}{c}1 \text { st } \\
\text { Millennium } \\
\text { BCE }\end{array}$} & Cimmerians & & \\
\hline & Srubnaya & \multicolumn{2}{|l|}{ VERT114 Bell Beaker } \\
\hline \multirow{2}{*}{$\begin{array}{c}\text { 2rd } \\
\text { Millennium } \\
\text { BCE }\end{array}$} & Srubnaya & & \\
\hline & Catacomb & \multicolumn{2}{|l|}{ VERT113 Corded Ware } \\
\hline \multirow{2}{*}{$\begin{array}{l}\text { 3th } \\
\text { Millennium } \\
\text { BCE }\end{array}$} & Yamnaya & & \\
\hline & Middle-Late CTCC & & \\
\hline \multirow{2}{*}{$\begin{array}{l}\text { 4th } \\
\text { Millennium } \\
\text { BCE }\end{array}$} & \multirow[b]{2}{*}{ Early CTCC } & Trypillia Hungary_LateC_EBA_Baden_Yamnaya 93\% & $\begin{array}{l}7 \% \\
\text { Yamnaya }\end{array}$ \\
\hline & & & \\
\hline
\end{tabular}

\section{Figure 4}

Ancestry and culture summary: Chronology of the different cultures that settled in Ukraine between the 4th and 1st millennium BCE; on the right, the ancestry components of the analysed individuals according to the qpAdm analyses.

\section{Supplementary Files}


This is a list of supplementary files associated with this preprint. Click to download.

- SupTables.xlsx

- SI.docx 